\title{
Biocompatibility and small protein permeability of hydrophilic-coated membrane dialyzer (NV) in hemodialysis patients: a pilot study
}

\author{
Hirotoshi Kodama ${ }^{1 *}$ (D) Akira Tsuji ${ }^{2}$, Akihiro Fujinoki ${ }^{1}$, Koujirou Ooshima ${ }^{2}$, Kaori Ishizeki ${ }^{2}$ and Tatsuo Inoue ${ }^{1}$
}

\begin{abstract}
Background: We evaluated the biocompatibility and small protein permeability of a newly developed hydrophilic-coated membrane dialyzer (NV) compared with conventional polysulfone dialyzer (APS) for hemodialysis (HD) therapy.
\end{abstract}

Methods: In a prospective crossover study, 11 maintenance HD patients (7 males; mean age $67.0 \pm 10.2$ years) received HD three times a week for 4 weeks with the NV membrane and then for another 4 weeks with the APS membrane. We evaluated the variation in several parameters including white blood cell (WBC) count and fibrinogen as indexes for biocompatibility. The plasma and dialysate concentrations of $\beta_{2}$-microglobulin $\left(\beta_{2}-M\right), a_{1}$-microglobulin $\left(a_{1}-M\right)$, and albumin were measured at baseline and after $4 \mathrm{~h}$ of each study treatment in order to assess the removal of small proteins.

Results: Reductions in the WBC count were seen with APS compared with NV at 60 min (NV $5.65 \pm 1.60$, APS 5. $17 \pm 1.65 \times 10^{3} / \mu \mathrm{L}, p<0.05$ ) and $240 \mathrm{~min}$ (NV $5.28 \pm 1.38$, APS $4.63 \pm 1.2 \times 10^{3} / \mu \mathrm{L}, p<0.005$ ) after the start of HD. With NV, we found significantly greater rates of variation of $\beta_{2}-\mathrm{M}$ (NV $45.5 \pm 1.2$, APS $40.1 \pm 1.2 \%, p<0.0001$ ), $a_{1}-\mathrm{M}$ (NV 41.2 \pm 9.9 , APS $34.2 \pm 18.5 \%, p<0.05$ ), and albumin (NV $31.6 \pm 7.8$, APS $18.1 \pm 6.5 \%, p<0.0001$ ) during HD than with APS. However, there were no significant differences in the removal of $\beta_{2}-M$ between the two dialyzers.

Conclusions: The clinical characteristics of NV may reveal an improved biocompatibility and a comparable efficiency in small protein removal as compared to those of APS.

Trial registration: Clinical effects of polysulfone membrane, NV-13U UMIN000011764

Keywords: Hemodialysis, Polysulfone, Hydrophilic-coated membrane, Biocompatibility, Small protein permeability

\section{Background}

Biocompatibility and middle molecule clearance of hemodialysis (HD) membranes affect the survival, morbidity, and quality of life of uremic patients undergoing maintenance HD therapy. In particular, malnutrition [1] and dialysis-related amyloidosis due to the reference middle molecule $\beta_{2}$-microglobulin $\left(\beta_{2}-\mathrm{M}\right)$ [2] are the major long-term complications. A polysulfone (PS) dialyzer is the mainstay of HD treatment because of its high performance. However, TORAYLIGHT ${ }^{\circ} \mathrm{NV}$ dialyzer (NV; Toray Industries, Inc., Tokyo, Japan) is expected to have different characteristics from the conventional PS due to its newly developed hydrophilic-coated membrane [3]. We therefore designed this prospective, crossover study to evaluate the biocompatibility and low-molecular-weight protein permeability (small protein permeability) of NV compared with a conventional PS (APS; ASAHIKASEI Industries, Inc., Tokyo, Japan).

\footnotetext{
* Correspondence: hiro25@jcom.zaq.ne.jp

${ }^{1}$ Division of Blood Purification Center, Kamifukuoka General Hospital, 931

Fukuoka, Fujimino, Saitama 356-0011, Japan

Full list of author information is available at the end of the article
} 

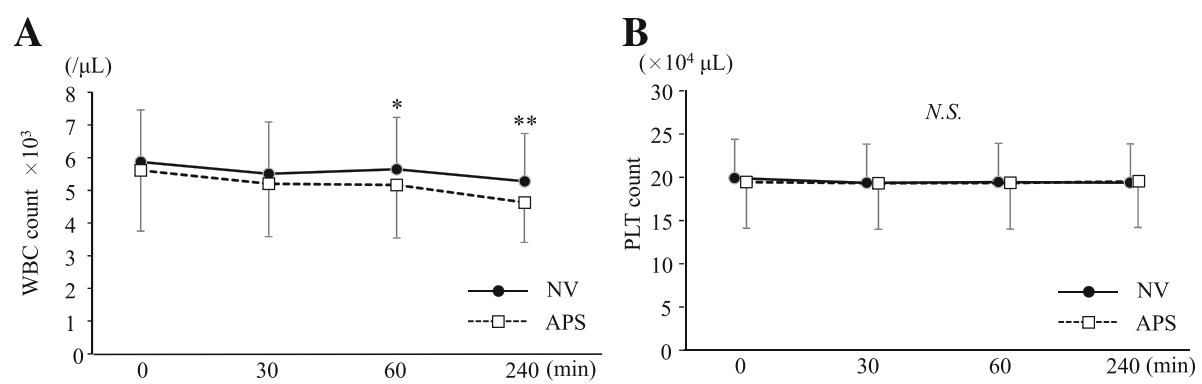

Fig 1 a WBC count and $\mathbf{b}$ PLT count. a Reduction in the white blood cell count were seen with the APS compared with the NV in 60 min $\left({ }^{*} p<0.05\right)$ and 240 min $\left({ }^{* *} p<0.005\right)$ after the start of HD. $\mathbf{b}$ There were no significant differences between NV and APS

\section{Methods}

Eleven maintenance HD patients ( 7 males; mean age $67.0 \pm 10.2$ years) on regular thrice-weekly HD treatment for $4 \mathrm{~h}$ were enrolled in the study after they had given their written informed consent. The underlying renal diseases were diabetes mellitus $(N=3)$, glomerulonephritis $(N=2)$, and unknown $(N=6)$. The mean duration of dialysis treatment was $48.6 \pm 45.0$ (range 15 to 258) months. All of the patients underwent 12 consecutive HD treatments with NV-13U $\left(1.3 \mathrm{~m}^{2}\right)$ and then switched with APS-13SA $\left(1.3 \mathrm{~m}^{2}\right)$ as a control after a 2-week wash-out period.

\section{Laboratory measurements}

The platelet (PLT) count, white blood cell (WBC) count, and hematocrit (Hct) were measured at the beginning of dialysis and after 30, 60, and $240 \mathrm{~min}$. To adjust for differences in the cell counts at the beginning of dialysis, we calculated the change ratio at each point using the following formula (1):

$$
\begin{aligned}
& \text { Change ratio } \mathrm{X} \min =100+100 \times\{(\text { Cell count } \times \min ) / \\
& \text { (Cell count } 0 \text { min) }-1 \text { \} }
\end{aligned}
$$

Cell count $0 \mathrm{~min}$ : Cell count at the beginning of dialysis

Cell count X min : Cell count after X minutes from the beginning of dialysis
We measured the urea nitrogen, serum creatinine, inorganic phosphorus, single-pool Kt/V [4], and hemoglobin levels; the removal rate of $\beta_{2}-\mathrm{M}$; and the rate of variation of $\beta_{2}-M, \alpha_{1}$-microglobulin $\left(\alpha_{1}-M\right)$, and albumin. We also measured the levels of platelet factor-4 (PF-4), $\beta$ thromboglobulin ( $\beta$-TG), fibrinogen/fibrin degradation products (FDP), D-dimer, and fibrinogen (FIB).

Dialysate samples were collected at the final two dialysis sessions at a speed of $1.0 \mathrm{~L} / \mathrm{h}$ using a fixed-quantity pump from the dialysate discharge line for 0-60, 60-180, and 180-240 min after the start of HD. On the assumption that each value measured before dialysis was $100 \%$, the results were evaluated for variability.

This study was conducted in accordance with the guidelines of the Declaration of Helsinki and approved by the ethics committees of Kamifukuoka General Hospital and National Defense Medical College Hospital.

\section{Statistical analyses}

The continuous values of variables are expressed as the mean \pm standard deviation or as the median (25th, 75th percentile). The comparative $p$ values for continuous variables were calculated by an analysis of variance (ANOVA). All of the statistical analyses were carried out using the JMP Pro Version 11.2.0 software program (SAS Institute, Cary, NC, USA), setting the significance level at a $p$ value of less than 0.05 .
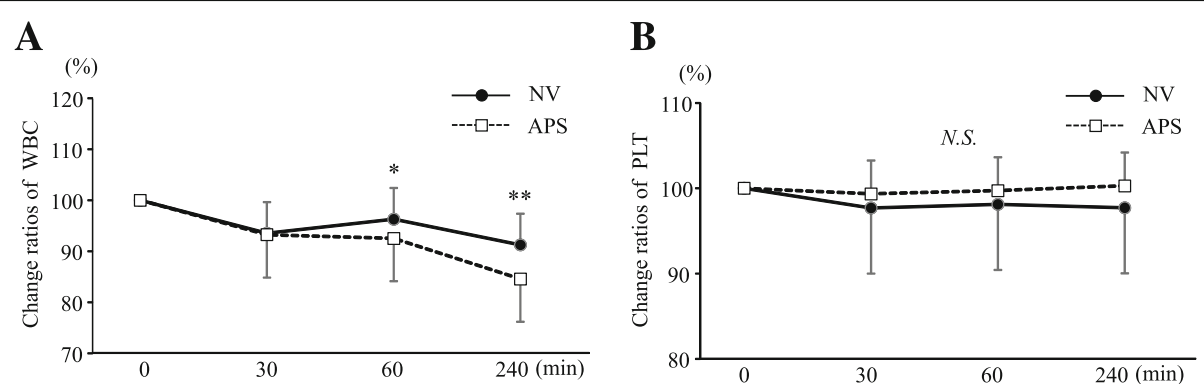

Fig 2 Change ratios of $\mathbf{a}$ WBC and $\mathbf{b}$ PLT. a The change ratios of the white blood cell count were significantly smaller with the NV than with the APS after 60 min $\left({ }^{*} p<0.01\right)$ and 240 min $\left({ }^{* *} p<0.05\right)$. b There were no significant differences between NV and APS 
Table 1 Clinical parameters of Hct, PF-4, $\beta-T G, F D P$, D-dimer, and change ratio of FIB

\begin{tabular}{|c|c|c|c|c|c|}
\hline & & 0 (min) & 30 (min) & 60 (min) & 240 (min) \\
\hline \multirow[t]{2}{*}{ Hct (\%) } & NV & $32.2 \pm 2.4$ & $31.4 \pm 2.7$ & $32.1 \pm 2.6$ & $34.9 \pm 3.3$ \\
\hline & APS & $32.0 \pm 2.4$ & $30.9 \pm 2.3$ & $31.5 \pm 2.3$ & $34.4 \pm 2.8$ \\
\hline \multirow[t]{2}{*}{ PF-4 (ng/mL) } & NV & $7.45 \pm 7.09$ & $29.2 \pm 13.5$ & $16.9 \pm 8.1$ & $12.3 \pm 7.4$ \\
\hline & APS & $6.77 \pm 3.69$ & $32.3 \pm 21.5$ & $17.3 \pm 6.9$ & $12.1 \pm 5.8$ \\
\hline \multirow[t]{2}{*}{$\beta-T G(n g / m L)$} & NV & $146 \pm 41$ & $128 \pm 42$ & $118 \pm 36$ & $104 \pm 39$ \\
\hline & APS & $135 \pm 33$ & $125 \pm 40$ & $119 \pm 36$ & $101 \pm 30$ \\
\hline \multirow[t]{2}{*}{$\mathrm{FDP}(\mu \mathrm{g} / \mathrm{mL})$} & NV & $2.0(2.0,2.0)$ & $2.0(2.0,2.0)$ & $2.0(2.0,2.0)$ & $2.0(2.0,3.0)$ \\
\hline & APS & $2.0(2.0,2.0)$ & $2.0(2.0,2.0)$ & $2.0(2.0,3.0)$ & $2.0(2.0,2.0)$ \\
\hline \multirow[t]{2}{*}{ D-dimer $(\mu \mathrm{g} / \mathrm{mL})$} & NV & $0.4(0.2,0.5)$ & $0.3(0.2,0.5)$ & $0.3(0.2,0.5)$ & $0.4(0.2,0.5)$ \\
\hline & APS & $0.4(0.4,0.5)$ & $0.3(0.2,0.5)$ & $0.3(0.2,0.5)$ & $0.3(0.2,0.5)$ \\
\hline \multirow[t]{2}{*}{ Change ratio of FIB (\%) } & NV & - & $98.6 \pm 2.8$ & $102 \pm 3$ & $117 \pm 8$ \\
\hline & APS & - & $97.8 \pm 3.3$ & $101 \pm 4$ & $116 \pm 11$ \\
\hline
\end{tabular}

FDP and D-dimer are expressed as medians with 25th-75th percentiles

$H c t$ hematocrit, PF-4 platelet factor-4, $\beta$-TG thromboglobulin, FDP fibrinogen/fibrin degradation products, FIB fibrinogen

\section{Results}

\section{Biocompatibility}

Reductions in the WBC count were seen with APS compared with NV at $60 \mathrm{~min}$ (NV $5.65 \pm 1.60$, APS $5.17 \pm$ $\left.1.65 \times 10^{3} / \mu \mathrm{L}, p<0.05\right)$ and $240 \mathrm{~min}(\mathrm{NV} 5.28 \pm 1.38$, APS $\left.4.63 \pm 1.20 \times 10^{3} / \mu \mathrm{L}, p<0.005\right)$ after the start of HD (Fig. 1). The PLT count was not markedly different between the two dialyzers. The change ratios of the WBC count were significantly smaller with NV than with APS after $60 \mathrm{~min}$ (NV $96.3 \pm 2.3$, APS $92.5 \pm 7.5 \%, p<0.01$ ) and $240 \mathrm{~min}$ (NV $91.3 \pm 2.3$, APS $84.6 \pm 11.3 \%, p<0.05)$ (Fig. 2).

No significant differences were seen in the levels of Hct, PF-4, $\beta$-TG, FDP, or D-dimer or in the change ratio of FIB between NV and APS (Table 1). There were also no significant differences in the urea nitrogen, creatinine, inorganic phosphorus, single-pool $\mathrm{Kt} / \mathrm{V}$, or hemoglobin values between the two dialyzers (Table 2).

\section{Small protein permeability}

The intradialytic removal of $\alpha_{1}-\mathrm{M}$ was greater with NV than with APS in the first hour (NV $15.7 \pm 4.0$, APS $10.7 \pm 3.0 \mathrm{mg}, p<0.001$ ) and last hour (NV $6.47 \pm 2.35$, APS $3.23 \pm 0.74 \mathrm{mg}, p<0.001$ ) as well as over the total HD session (NV $32.0 \pm 11.3$, APS $17.7 \pm 6.3 \mathrm{mg}, p<0.0001$ )

Table 2 Clinical parameters

\begin{tabular}{llll}
\hline & NV & APS & $p$ \\
\hline Urea nitrogen $(\mathrm{mg} / \mathrm{dL})$ & $61.1 \pm 13.8$ & $62.1 \pm 11.7$ & N.S. \\
Creatinine $(\mathrm{mg} / \mathrm{dL})$ & $9.98 \pm 2.39$ & $9.87 \pm 2.23$ & N.S. \\
Phosphorus $(\mathrm{mg} / \mathrm{dL})$ & $4.84 \pm 1.31$ & $4.95 \pm 1.25$ & N.S. \\
Single-poor Kt/N & $1.55 \pm 0.22$ & $1.63 \pm 0.21$ & N.S. \\
Hemoglobin $(\mathrm{g} / \mathrm{dL})$ & $10.5 \pm 0.9$ & $10.3 \pm 0.7$ & N.S. \\
\hline
\end{tabular}

N.S. not significant
(Fig. 3). In addition, the removal of albumin was greater with NV than with APS in the first hour (NV $340 \pm 125$, APS $198 \pm 59 \mathrm{mg}, p<0.001$ ) and last hour (NV $109 \pm 46$, APS $32.0 \pm 3.8 \mathrm{mg}, p<0.001)$ as well as over the total HD session (NV $693 \pm 283$, APS $249 \pm 90 \mathrm{mg}, p<0.001$ ) (Fig. 4). There were no significant differences in the removal of $\beta_{2}$-M between the two dialyzers (Fig. 5). However, the rates of variation during HD were greater with $\mathrm{NV}$ than with APS for $\beta_{2}$-M (NV $45.5 \pm 1.2$, APS $40.1 \pm 1.2 \%, p<$ $0.001), \alpha_{1}-\mathrm{M}$ (NV $41.2 \pm 9.9$, APS $34.2 \pm 18.5 \%, p<0.05$ ), and albumin (NV $31.6 \pm 7.8$, APS $18.1 \pm 6.5 \%, p<0.001$ ).

\section{Discussion}

The PS membrane has shown a very good biocompatibility due to its superior removal performance for small-molecule proteins such as $\beta_{2}-\mathrm{M}$, high clearance of small-molecule solutes such as urea and creatinine [5], and low rate of complications such as leukopenia or disorders associated with the complement system [6]. Therefore, the PS membrane is widely used for HD treatment in Japan [7]. The NV membrane was developed as an alternative to PS. With NV, the mobility of water adjacent to the membrane surface is enhanced using a new hydrophilic polymer to reform the membrane surface. NV has antithrombotic activity and less platelet and leukocyte activation than PS, as well as the ability to sufficiently remove small solutes and lowmolecular-weight proteins [3]. Further, NV induces less interleukin- 6 activity, which may reduce the risk of erythropoiesis-stimulating agent hyporesponsiveness [8]. In this study, we evaluated the biocompatibility and protein permeability of $\mathrm{NV}$ compared with those of APS as a conventional PS.

The variation of several parameters such as the WBC, PLT, Hct and levels of PF-4, $\beta$-TG, FDP, D-dimer, and 

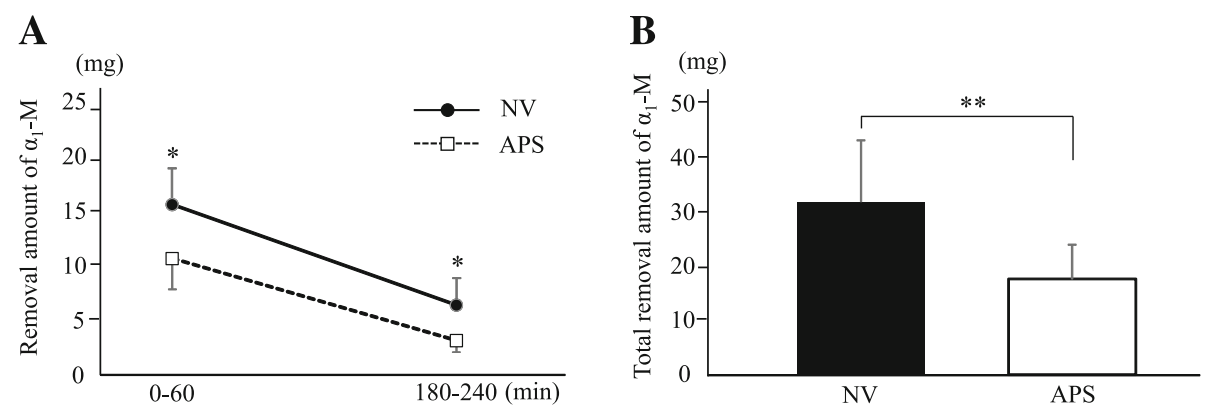

Fig 3 a Removal amount of $a_{1}-M$ and $\mathbf{b}$ total removal amounts of $a_{1}-M$. Compared to APS. ${ }^{*} p<0.001$ and ${ }^{* *} p<0.0001$ by paired $t$ test. $a_{1}-M a_{1}$-microglobulin

FIB were compared to evaluate the biocompatibility of NV. PF4 and $\beta-T G$ are substances contained within platelet $\alpha$-granules, and changes in platelet PF4 or $\beta$-TG levels are useful indicators of platelet degranulation and activation during dialysis [9]. However, there were no significant changes observed in the coagulation factor values with either dialyzer. Although acute leukopenia is known to occur in all patients during the first $30 \mathrm{~min}$ of HD treatment [10], our study showed no reduction in the WBC during dialysis with either dialyzer. The platelet count usually decreases 5 to $15 \%$ at 15 to $30 \mathrm{~min}$ from the start of HD and recovers to the normal level by the end of the dialysis session $[9,11]$. Oshihara [3] reported that the numbers of platelets adhering to the membrane surface were lower with NV than with the conventional PS (CX) membrane. In our results, the variations in the platelet counts with both membranes were stable, suggesting that both may prevent HD-associated thrombocytopenia.

It is known that WBC adhesion occurs with protein adsorption, and FIB adsorption promotes PLT adhesion on the dialysis membrane surface by blood exposure in HD treatment [12]. Platelet-derived microparticles are released from activated platelets during HD [13]. Although the biocompatibility of PS is generally regarded to be good, there are some reports that the PS dialyzer caused PLT depletion $[14,15]$. On the other hand, the NV membrane has been reported that it not only inhibits the adhesion of blood components such as PLT and FIB but also acts as a highly biocompatible membrane to reduce blood cell stimulation because it is developed as a new hydrophilic polymer membrane [3]. With NV, the reduction rate of the WBC count was inhibited to a greater degree than with APS. However, the change ratio of FIB showed no significant difference between NV and APS. These results suggest that NV improves the biocompatibility compared with APS.

Dialysis-related amyloidosis is a major complication with long-term HD treatment and greatly affects patients' quality of life. The removal of $\beta_{2}-\mathrm{M}$ is therefore important to avoid this complication. However, there have been no reports of the small protein permeability of NV. We therefore designed this prospective crossover study to evaluate whether or not NV offers any advantages over APS. We found that the intradialytic removal of $\alpha_{1}-\mathrm{M}$ and albumin but not $\beta_{2}-\mathrm{M}$ was significantly greater with $\mathrm{NV}$ than with APS. The rates of variation in the $\beta_{2}-M$ and $\alpha_{1}-M$ levels during $H D$ with NV were higher than with APS, but the differences were small. These results suggest that the removal performance of $\beta_{2}-\mathrm{M}$ with NV and APS should be almost equivalent.
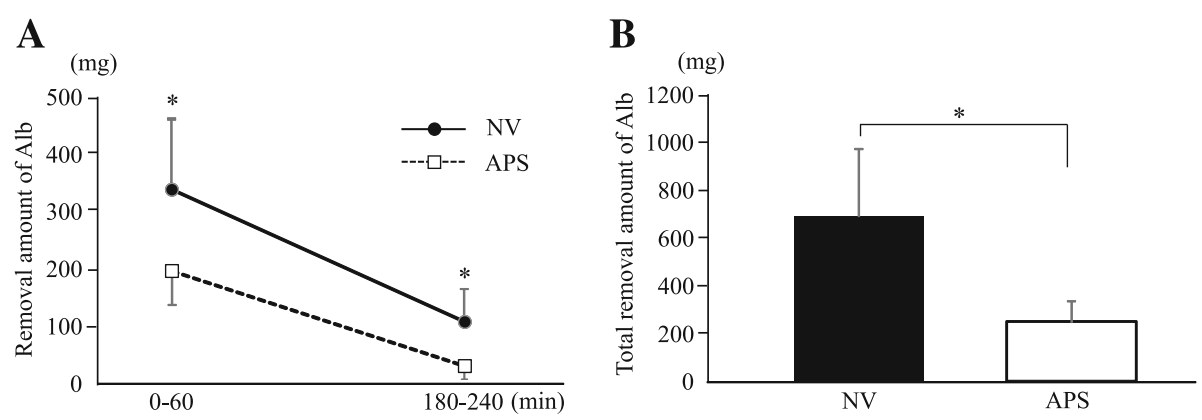

Fig 4 a Removal amount of Alb and $\mathbf{b}$ total removal amounts of Alb. Compared to APS. ${ }^{*} p<0.0001$ by paired $t$ test. Alb albumin 
A

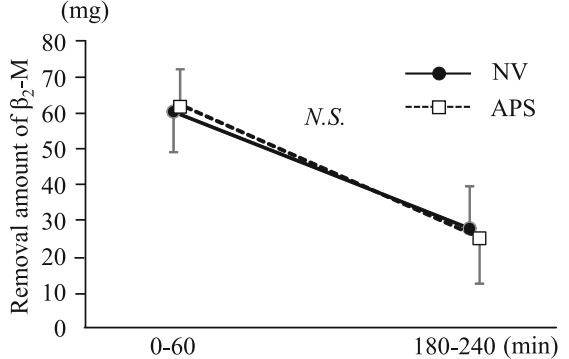

B

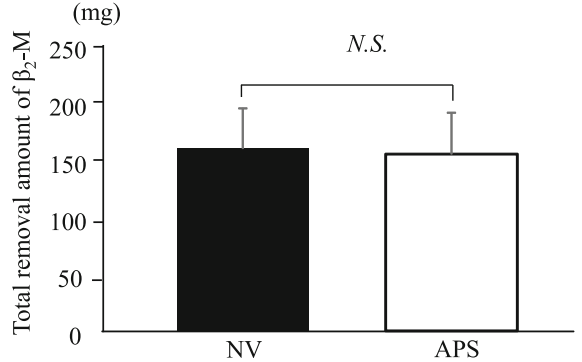

Fig 5 a Removal amount of $\beta_{2}-M$ and $\mathbf{b}$ total removal amounts of $\beta_{2}-M$. There were no significant differences between NV and APS. $\beta_{2}-M \beta_{2}$-microglobulin

In addition, since the decrease in the serum albumin may cause low muscle mass [16], malnutrition [17], and mortality risk [18], it is important to select dialyzers with consideration of the laboratory data. NV removed significantly greater albumin than APS. Therefore, we should be cautious when using the NV membrane in elderly or malnourished patients, although the amount of albumin removed was less than $1 \mathrm{~g}$ (NV $693 \pm 283$, APS $249 \pm 90 \mathrm{mg}$ ) in either dialyzer.

Because this was a short-term, prospective, crossover study involving only a few cases, large-scale and long-term future trials are necessary to corroborate our findings.

\section{Conclusions}

We designed this crossover study of the NV and APS membranes to evaluate the biocompatibility and small protein removal ability. The clinical characteristics of NV may reveal an improved biocompatibility and a comparable efficiency in small protein removal as compared to APS.

\section{Abbreviations}

Alb: Albumin; FDP: Fibrinogen/fibrin degradation products; FIB: Fibrinogen; Hct: Hematocrit; HD: Hemodialysis; PF-4: Platelet factor-4; PLT: Platelet; PS: Polysulfone; WBC: White blood cell; $a_{1}-M$ : $a_{1}-$ Microglobulin;

$\beta_{2}-$ M: $\beta_{2}$-Microglobulin; $\beta$-TG: $\beta$-Thromboglobulin

\section{Acknowledgements}

The authors thank all the staff members working at the Division of Blood Purification Center, Kamifukuoka General Hospital and Department of Blood Purification Therapy, National Defense Medical College Hospital.

\section{Funding}

This study has received research funds from the Foundation for Promotion of Defense Medicine.

\section{Availability of data and materials}

Data sharing is not applicable to this manuscript.

\section{Authors' contributions}

HK designed the study, collected the clinical data, analyzed the data, and wrote the manuscript. AT designed the study and wrote the manuscript. AF collected the clinical data. $\mathrm{Kl}, \mathrm{KO}$, and $\mathrm{TI}$ participated in its design and coordination. All authors read and approved the final manuscript.
Ethics approval and consent to participate

This study was conducted in accordance with the guidelines of the Declaration of Helsinki and approved by the ethics committees of Kamifukuoka General Hospital (approval number 24-01) and National Defense Medical College Hospital (approval number 939). Written informed consent was obtained from the patients.

\section{Consent for publication}

We have also obtained the consent to publish from the participant to report the individual patient data.

\section{Competing interests}

The authors declare that they have no competing interests.

\section{Publisher's Note}

Springer Nature remains neutral with regard to jurisdictional claims in published maps and institutional affiliations.

\section{Author details}

${ }^{1}$ Division of Blood Purification Center, Kamifukuoka General Hospital, 931 Fukuoka, Fujimino, Saitama 356-0011, Japan. ²Department of Blood Purification, National Defense Medical College Hospital, 3-2 Namiki, Tokorozawa, Saitama 359-8513, Japan.

Received: 8 November 2016 Accepted: 6 July 2017

Published online: 16 October 2017

\section{References}

1. Kalantar-Zadeh K, Kopple JD, Block G, Humphreys MH. A malnutritioninflammation score is correlated with morbidity and mortality in maintenance hemodialysis patients. Am J Kidney Dis. 2001;38:1251-63.

2. Gejyo F, Yamada T, Odani S, Nakagawa Y, Arakawa M, Kunitomo T, et al. A new form of amyloid protein associated with chronic hemodialysis was identified as beta 2-microgulobulin. Biophys Res Commun. 1985;129:701-6.

3. Oshihara W, Ueno Y, Fujieda H. A new polysulfone membrane dialyzer, NV, with low-founding and antithrombotic properties. Contrib Nephrol. 2017:189:222-9.

4. Shinzato T, Nakai S, Fujita Y, Takai I, Morita E, Nakane K, Maeda K. Determination of Kt/N and protein catabolic rate using pre- and postdialysis blood urea nitrogen concentrations. Nephron. 1994;67:280-90.

5. Fukuda M, Miyazaki M, Uezumi S, Yoshida M. Design and assessment of the new APS dialyzer (APS-SA series). J Artifs Organs. 2006;9:192-8.

6. Yamashita S, Mochizuki A, Nakazaki T, Seita Y, Sawamoto J, Endo F, et al. A new blood compatible and permselective hollow fiber membrane for hemodialysis. ASAIO J. 1996;42(6):1019-26.

7. Nakai S, Suzuki K, Masakane I, et al. Overview of regular dialysis treatment in Japan (as of 31 December 2009). Ther Apher Dial. 2010;14:505-40.

8. Kakuta T, Komaba H, Takagi N, Takahashi Y, Suzuki H, et al. A prospective multicenter randomized controlled study on interleukin-6 removal and induction by a new hemodialyzer with improved biocompatibility in hemodialysis patients: a pilot study. Ther Apher Dial. 2016;20(6):569-78. 
9. Daugirdas JT, Bernardo AA. Hemodialysis effect on platelet count and function and hemodialysis-associated thrombocytoperia. Kidney Int. 2012;82:147-57

10. Kaplow LS, Goffinet JA. Profound neutropenia during the early phase of hemodialysis. JAMA. 1968;203:1135-7.

11. Amato M, Salvadori M, Bergesio F, Messeri A, Filimberti E, Morfini M. Aspects of biocompatibility of two different dialysis membranes: cuprophane and polysulfone. Int J Artif Organs. 1988;11:175-80.

12. Reginald G. Mason, Hanson Y.K. chuang, S. Fazal Mohammad. Extracorporeal thrombogenesis: mechanisms and prevention. replacement of renal function by dialysis. 1983: 186-200.

13. Daniel L, Fakhouri F, Joly D, et al. Increase of circulating neutrophil and platelet microparticles during acute vasculitis and hemodialysis. Kidney Int. 2006;69:1416-23.

14. Kobari E, Terawaki $H$, Takahashi $Y$, et al. Dialyzer-related thrombocytopenia due to a polysulfone membrane. Intern Med. 2016;55:965-8.

15. Deprada L, Lee J, Gillespie A, Benjamin J. Thrombocytopenia associated with one type of polysulfone hemodialysis membrane: a report of 5 cases. Am J Kidney Dis. 2013;61:131-3.

16. Yasui S, Shirai Y, Tanimura M, Matsuura S, Saito Y, Miyata K. Prevalence of protein-energy wasting (PEW) and evaluation of diagnostic criteria in Japanese maintenance hemodialysis patients. Asia Pac J Clin Nutr. 2016;25(2):292-9.

17. Pifer TB, McCullough K, Port FK, Goodkin DA, Maroni BJ, Held PJ, et al. Mortality risk in hemodialysis patients and changes in nutritional indicators: DOPPS. Kidney Int. 2002;62:2338-245.

18. Dwyer JT, Larive B, Leung J, Rocco MV, Greene T, Burrowes J, et al. Are nutritional status indicators associated with mortality in hemodialysis (HEMO) study? Kidney Int. 2005:68:1766-76.

\section{Submit your next manuscript to BioMed Central and we will help you at every step:}

- We accept pre-submission inquiries

- Our selector tool helps you to find the most relevant journal

- We provide round the clock customer support

- Convenient online submission

- Thorough peer review

- Inclusion in PubMed and all major indexing services

- Maximum visibility for your research

Submit your manuscript at www.biomedcentral.com/submit 